Claremont Colleges

Scholarship@ Claremont

All HMC Faculty Publications and Research

HMC Faculty Scholarship

$1-1-2002$

\title{
Time-Dependent Density Functional Theory Investigation of the Ground and Excited States of Coumarins 102, 152, 153, and 343
}

Robert J. Cave

Harvey Mudd College

Edward W. Castner Jr.

Rutgers University

\section{Recommended Citation}

Cave, R.J; Castner, E. W. Jr. “Time-Dependent Density Functional Theory Investigation of the Ground and Excited States of Coumarins 102, 152, 153, and 343," J. Phys. Chem. A 2002, 106, 12117. DOI: 10.1021/jp026718d

This Article is brought to you for free and open access by the HMC Faculty Scholarship at Scholarship @ Claremont. It has been accepted for inclusion in All HMC Faculty Publications and Research by an authorized administrator of Scholarship @ Claremont. For more information, please contact scholarship@cuc.claremont.edu. 


\title{
Time-Dependent Density Functional Theory Investigation of the Ground and Excited States of Coumarins 102, 152, 153, and 343
}

\author{
Robert J. Cave* \\ Department of Chemistry, Harvey Mudd College, Claremont, California 91711 \\ Edward W. Castner, Jr.* \\ Department of Chemistry and Chemical Biology, Rutgers University, 610 Taylor Road, \\ Piscataway, New Jersey 08854-8087
}

Received: August 8, 2002; In Final Form: October 14, 2002

\begin{abstract}
We present calculations of various properties of the ground and excited electronic states of coumarins 102, 152, 153, and 343. Using density functional theory (DFT) and time-dependent density functional theory (TDDFT), we examine the excitation energies to the $S_{1}$ and $S_{2}$ states, the ground and excited-state dipole moments, and the lowest ionization potentials of these coumarins. In the case of C153, we locate two distinct $\mathrm{S}_{0}$ minima due to differing conformations of the julolidyl ring structure and compare properties for the syn and anti conformers. For C343, we examine the possibility of proton transfers in the ground and $\mathrm{S}_{1}$ states of the system. We find that (1) DFT tends to overestimate the ground-state dipole moments in these systems, (2) excellent agreement is obtained between TDDFT and experimental vertical excitation energies, (3) TDDFT and CIS yield similar estimates of the dipole moment change between the $S_{0}$ and $S_{1}$ states, both of which are in the range of previous experimental estimates, (4) in each case, the $S_{2}$ state is at least $0.5 \mathrm{eV}$ above the $\mathrm{S}_{1}$ state for the ground-state geometry, and (5) proton transfer is not likely in the ground state of C343 but is only $0.18 \mathrm{eV}$ higher in energy in the $\mathrm{S}_{1}$ state. We also compare the DFT/TDDFT results with RHF/CIS, MP2, and INDO S/CI results. We find good agreement between MP2 and experimental ground-state dipole moments and good agreement between INDO S/CI and TDDFT gas-phase excitation energies.
\end{abstract}

\section{Introduction}

The 7-aminocoumarins (see Scheme 1) are robust chromophores that are used in a broad range of applications. The excited-state properties of more than 10 different 7-aminocoumarins in solution have been well characterized for use as laser dyes. ${ }^{1}$ The strong absorption cross sections and high fluorescence quantum yields, combined with low cross sections for other photophysical processes, led to wide use of 7-aminocoumarins for laser action from the near-ultraviolet $(350 \mathrm{~nm})$ to the middle of the visible light spectrum $(500 \mathrm{~nm}){ }^{1}$

As dye lasers are increasingly replaced by solid-state laser technologies, the coumarins have found an increasing number of applications in probing the dynamics of condensed-phase environments. The properties that cause the 7-aminocoumarins to be effective laser dyes are the very same ones that make them desirable for use as fluoroprobes: (1) they have strong radiative rates, (2) they are fairly rigid, and (3) they possess strong solvatochromism. The relative rigidity of the coumarin framework, especially the closed-ring amine system (julolidyl group) present for coumarins 102, 153, and 343, means that the coumarins are good probes of local friction in solutions and in more-complex environments when using time-resolved fluorescence polarization anisotropies. ${ }^{2-11}$ For investigating proteins and amphiphiles, coumarins can be used to probe both hydrophilic environments (by using water-soluble C343 and sparingly soluble C102) and hydrophobic regions (by using water-insoluble $\mathrm{C} 153)$.

* Corresponding author. E-mail: Robert_Cave@Hmc.edu. E-mail: castner@rutchem.rutgers.edu.

\section{SCHEME 1}<smiles>Cc1cc(=O)oc2c3c4c(cc12)CCCN4CCC3</smiles>

coumarin 102

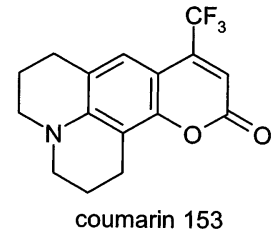<smiles>CN(C)c1ccc2c(C(F)(F)F)cc(=O)oc2c1</smiles>

coumarin 152<smiles>O=C(O)c1cc2cc3c4c(c2oc1=O)CCCN4CCC3</smiles>

coumarin 343
The strong solvatochromism of the coumarins results from the substantial increase in the permanent dipole moment on going from the ground to the excited state. This causes a bathochromic (red) shift in the fluorescence that depends linearly on the empirically determined solvent polarity. This red shift in the emission can exceed $5000 \mathrm{~cm}^{-1}$ for the most-polar solvents (e.g., water), which is more than $20 \%$ of the total excited-state energy. Using time-resolved emission methods such as femtosecond fluorescence upconversion spectroscopy, this emission red shift can be time-resolved and is called the timedependent fluorescence Stokes shift (TDFSS). ${ }^{12-19}$

The TDFSS method has been well characterized for use in probing the dynamics of the liquid state. ${ }^{12-20}$ Many of the most successful studies have used 7-aminocoumarins as probes of 
the TDFSS. More recently, the 7-aminocoumarins have been used to probe dynamic fluctuations about a charge-transfer excited state for a wide variety of condensed-phase systems. These include polymers, ${ }^{11,21}$ reverse and normal micelles, ${ }^{3-5,22,23}$ semiconductor surfaces, ${ }^{24}$ zeolites, ${ }^{25}$ sol-gels, ${ }^{7}$ vesicles, ${ }^{6}$ proteins, ${ }^{26}$ cyclodextrins, ${ }^{9}$ and molten salts. ${ }^{27,28}$

The coumarins can be detected on a single-molecule level ${ }^{29}$ but will find only limited applications in this area because they photoreact after prolonged cycles of excitation/emission. Though a less than optimal choice for single-molecule spectroscopy, 7-aminocoumarins have proven to be ideal probes for certain electron-transfer processes. ${ }^{30,31}$ In particular, these coumarins have been widely used as excited-state electron acceptors to study electron transfer from electron-donating solvents such as aromatic amines. ${ }^{7,32-37}$ Another application of the 7-aminocoumarins, especially the 3-carboxylic acid derivatives, has been as photosensitizing dyes for wide band gap semiconductors such as $\mathrm{TiO}_{2}$ and $\mathrm{ZnO} .^{24,38-42}$ Excited-state proton-transfer reactions have also been widely studied using 7 -aminocoumarins. ${ }^{43-48}$

We have recently published a theoretical study of coumarins 151 and $120.4^{49}$ These coumarins have been used in a variety of applications but are small enough to admit treatment with a wide range of electronic structure techniques. We compared the results from time-dependent density functional theory (TDDFT), ${ }^{50-52}$ using pure GGAs and hybrid functionals, with complete active space self-consistent field (CASSCF) results, CASPT2 (both single- and multistate versions), ${ }^{53-55}$ configuration interaction singles (CIS), and INDO S/CI. ${ }^{56,57}$ We found that (1) TDDFT gave excellent agreement with experimental $S_{1} \leftarrow S_{0}$ excitation energies for C151 and C120, (2) TICT formation is unlikely upon photexcitation for gas-phase $\mathrm{C} 151$, (3) there is a greater tendency toward a planar amine group for the $S_{1}$ state than for the ground state of C151, (4) DFT tended to overestimate the ground-state dipole moments, and (5) TDDFT results for C151water complexes are in good agreement with the experimental results of Topp and co-workers. ${ }^{58,59}$ On the basis of the success of DFT and TDDFT for C120 and C151, we apply these methods here to a series of coumarins that have been important in probing solvent dynamics.

In the present contribution, we survey various ground- and excited-state properties of C102, C152, C153, and C343 using DFT and TDDFT on the basis of the PBE0 functional. ${ }^{60}$ For comparison purposes, we also present results based on RHF, MP2, and INDO/S for the ground-state dipole moments of each species and CIS and INDO S/CI for excited-states properties. In the case of $\mathrm{C} 153$, we treat several ground-state conformers (syn and anti conformers of the julolidyl ring system) and investigate how the ground- and excited-state properties vary with conformation. For C343, we also examine the ground- and excited-state energies of various placements of the ionizable proton on the carbonyl group.

The remainder of the article is organized as follows. In the following section, we outline the theoretical methods used to treat the coumarins. In section III, we present our results, and in section IV, the results are discussed. In the final section, we offer concluding remarks.

\section{Methods}

All ab initio calculations use the $6-311 \mathrm{G}(\mathrm{d}, \mathrm{p})$ basis set. ${ }^{61,62}$ In our previous work, this basis was shown to be adequate for the calculation of properties associated with the ground and first few excited states of $\mathrm{C} 151$ and $\mathrm{C} 120 .{ }^{49}$

The ground-state geometries of C102, C152, C153, and C343 were obtained from optimizations using DFT with the B3LYP

\section{SCHEME 2}

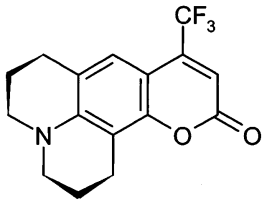

syn

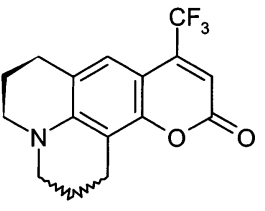

anti functional ${ }^{63}$ (geometries provided in Supporting Information). For $\mathrm{C} 152$, the $-\mathrm{N}\left(\mathrm{CH}_{3}\right)_{2}$ group was nearly planar and coplanar with the ring to which it was attached. For C102, C153, and $\mathrm{C} 343$, the $\mathrm{S}_{0}$ ground states were syn with respect to the $\mathrm{N}$ of the unsaturated rings. We separately located two anti structures for $\mathrm{C} 153$ (see Scheme 2). However, the two anti structures are essentially degenerate, and results are presented for only one of the two. For C343, we also explored different placements of the carboxyl proton (see Results).

Based on the $\mathrm{S}_{0}$ geometries for each coumarin, we performed DFT calculations using the PBE0 functional ${ }^{60}$ to obtain groundstate dipole moments and relative energies of ground-state conformers. In addition, TDDFT calculations were performed using the PBE0 functional within the adiabatic approximation. Dipole moments for TDDFT excited states were estimated using a finite field calculation $( \pm 0.001 \mathrm{au})$ and TDDFT excitation energies. ${ }^{49}$ For C343, we also report polarized continuum model $^{64-66}$ (PCM) calculations for the various ground-state conformers, with the solvent dielectric chosen to model chloroform.

For comparison purposes, we also performed RHF and MP2 calculations for the $S_{0}$ states and CIS calculations for the $S_{1}$ and $S_{2}$ states. CIS dipole moments are calculated analytically.

All DFT, TDDFT, RHF, MP2, and CIS calculations and all geometry optimizations were performed using Gaussian 98 (revisions A.9 and A.11). ${ }^{67}$

We also performed calculations using the INDO S/CI method of Zerner and co-workers. ${ }^{56,57}$ The results presented below are based on the Zerner group code (though similar results are obtained when the INDO S/CI method is used within the G98 code). ${ }^{49}$

\section{Results}

The results for the four coumarins are grouped together according to the molecular property of interest. In addition, we present previous results for the given property and for coumarin where available. For convenience, tables of the properties for each of the coumarins are reported in the Supporting Information.

Ground-state dipole moments based on RHF, DFT (using the PBE0 functional), and MP2 are reported in Table 1. We also present values from previous semiempirical methods (AM1, ${ }^{68}$ PM $3^{69,70}$ ) and density functional theory results. Our PBE0 dipole moments tend to be higher than those from experiment, with the greatest differences occurring for $\mathrm{C} 152$ and $\mathrm{C} 343$. The syn and anti conformers of $\mathrm{C} 153$ have similar dipole moments, with the anti conformer values being about $0.4 \mathrm{D}$ higher than those of the syn conformer. RHF dipole moments are similar to the PBE0 results, whereas MP2 represents an improvement relative to RHF results in each case except for $\mathrm{C} 102$. The largest errors relative to experiment for RHF once again occur for $\mathrm{C} 152$ and C343.

In our previous study of $\mathrm{C} 151$ and $\mathrm{C} 120$, we noted significant differences between the experimental and our theoretical groundstate dipole, with the experimental dipole moment significantly 
TABLE 1: Comparison of Ground-State Dipole Moment Values (D)

\begin{tabular}{llllr}
\hline method & C152 & \multicolumn{1}{c}{ C153 } & C102 & C343 \\
\hline expt $^{a} 71$ & 5.71 & 6.55 & 6.98 & 9.86 \\
PM3 $^{71}$ & 4.63 & 5.21 & 5.73 & 9.33 \\
AM1 $^{73}$ & 6.32 & 6.68 & 6.43 & 10.41 \\
AM1 $^{77}$ & 6.27 & 6.42 & & \\
DFT $^{72}$ & & $8.1 / 7.0$ & & \\
INDO $^{76}$ & & 7.4 & & \\
RHF $^{76}$ & & 6.1 & & \\
INDO & 6.48 & $6.56 / 7.52^{b}$ & 6.98 & 10.10 \\
RHF & 6.97 & $6.94 /-$ & 7.16 & 12.04 \\
PBE0 & 7.18 & $7.24 / 7.61^{c}$ & 6.81 & 11.76 \\
MP2 & 6.46 & $6.43 / 6.81^{c}$ & 5.99 & 10.66
\end{tabular}

${ }^{a}$ Measured in $\mathrm{CHCl}_{3}$ solvent. ${ }^{b}$ The first entry uses the gas-phase $\mathrm{O}$ beta value $(-54.0)$, and the second uses the solution-phase value $(-34.0){ }^{c}$ The first entry is for the syn structure, and the second is for the anti structure.

TABLE 2: Comparison of $S_{1} \leftarrow S_{0}$ Transition Energies (eV)

\begin{tabular}{lllll}
\hline \multicolumn{1}{c}{ source } & $\mathrm{C} 152$ & \multicolumn{1}{c}{$\mathrm{C} 153$} & $\mathrm{C} 102$ & $\mathrm{C} 343$ \\
\hline AM13 & 3.21 & 3.06 & 3.32 & 3.13 \\
solvatochromic $\nu_{\text {vert }}{ }^{78}$ & & 3.38 & 3.62 & \\
optical $\nu_{0-0}{ }^{72}$ & & 3.15 & & \\
optical/DFT $\nu_{\text {vert }}{ }^{72}$ & & 3.41 & & \\
DFT & & & \\
optical $\nu_{0-0}{ }^{79}$ & $3.17 / 3.37^{a}$ & & \\
optical $\nu_{\text {vert }}{ }^{79}$ & 3.56 & 3.21 & & \\
solution $\nu_{\text {vert }}$ & $3.15^{b}$ & $2.93^{c}$ & $3.18^{b}$ & $3.03^{d}$ \\
INDO S/CI & 3.77 & $3.66 / 3.51^{e}$ & 3.73 & 3.77 \\
PBE0/TDDFT & 3.58 & $3.41 / 3.41^{f}$ & 3.70 & 3.45 \\
CIS & 4.76 & 4.59 & 4.81 & 4.45
\end{tabular}

${ }^{a}$ The first entry is for S/CI, and the second is for MRSDCI. ${ }^{b}$ Spectral peak in methanol solvent. ${ }^{c}$ Spectral peak in ethanol solvent. ${ }^{d}$ Spectral peak in basic methanol solvent. ${ }^{e}$ The first entry uses the gas-phase $\mathrm{O}$ beta value $(-54.0)$, and the second uses the solution-phase value $(-34.0) .{ }^{f}$ The first entry is for the syn structure, and the second is for the anti structure.

smaller than the calculated value. We suggested that the difference might arise from partial twisting about the 7-amino bond in solution and demonstrated that twisting leads to an overall dipole moment decrease for $\mathrm{C} 151$. A similar effect may be operative for $\mathrm{C} 152$. $\mathrm{C} 153$ and $\mathrm{C} 102$ prohibit twisting by the presence of the julolidyl rings, and we note better agreement between our calculated dipole values and the experimental values of Moylan. ${ }^{71} \mathrm{C} 343$ similarly restricts twisting about the 7-amino bond, but our theoretical estimates for the dipole moment are once again too high. It is possible that nonplanar conformations are adopted by the carboxyl group in solution, which could lead to smaller dipole moments.

As noted previously, the PM3 $3^{69,70}$ dipole moments are uniformly lower than the experimental values. AM168 and INDO methods do a remarkably good job of estimating the dipole moment for these systems, which are similar to the MP2 results and are in better agreement with experiment than either the RHF or PBE0 results. The previous DFT results for $\mathrm{C} 153^{72}$ are comparable to our PBE0 results. Differences in the dipole moment for the previous DFT results depend on whether the S/CI or SDCI method of Grimme is used.

Excitation energies to the $S_{1}$ state of the various coumarins are presented in Table 2. Except for the entry "solution $v_{\text {vert }}$ ", the experimental values are either gas-phase values or extrapolations to gas-phase values from solution-phase results for direct comparison with our gas-phase results. Since our excitation energies are computed at the $S_{0}$ equilibrium geometry, they are most directly comparable with vertical transition energies $\left(v_{\text {vert }}\right)$. We find that the PBE0/TDDFT results are in excellent agree-
TABLE 3: Comparison of Dipole Moment Differences between the $S_{0}$ and $S_{1}$ States (D)

\begin{tabular}{|c|c|c|c|c|}
\hline source & C152 & C153 & C102 & C343 \\
\hline microwave $^{80}$ & $5.1 / 6.1^{a}$ & $4.9 / 5.4^{a}$ & $3.0 / 3.8^{a}$ & \\
\hline solvatachromism $^{77}$ & $9.23^{a}$ & $7.20^{a}$ & & \\
\hline solvatachromism $^{81}$ & $2.16^{a}$ & $2.22^{a}$ & $2.11^{a}$ & \\
\hline DC photocurrent ${ }^{82}$ & & $9.0^{a}$ & & \\
\hline electrooptic ${ }^{75}$ & & $4.40-7.00^{a}$ & & \\
\hline $\mathrm{AM} 1^{73}$ & $6.79^{a}$ & $6.96^{a}$ & $3.66^{a}$ & $5.29^{a}$ \\
\hline INDO S/CI ${ }^{76}$ & & 7.5 & & \\
\hline $\mathrm{CIS}^{76}$ & & 3.7 & & \\
\hline $\mathrm{DFT}^{72}$ & & $15.3 / 10.7^{a}$ & & \\
\hline $\mathrm{CIS}^{75}$ & & 4.88 & & \\
\hline INDO S/CI & 4.27 & $3.94 / 7.91^{b}$ & 2.07 & 4.36 \\
\hline PBE0/TDDFT & 5.9 & 5.8 & 5.2 & 4.1 \\
\hline CIS & 3.82 & 4.03 & 2.78 & 3.00 \\
\hline
\end{tabular}

${ }^{a}$ Collinearity assumed. ${ }^{b}$ The first entry uses the gas-phase $\mathrm{O}$ beta value $(-54.0)$, and the second uses the solution-phase value (-34.0). ment with the experimental vertical transition energies and are in substantially better agreement than are the CIS results. The previous AM1 values ${ }^{73}$ reproduce the trends in relative excitation energies but tend to be about $0.3 \mathrm{eV}$ too low, where comparisons are possible.

We were unable to locate a gas-phase value for the C343 vertical transition energy in the literature. To compare the results for all four systems, we use the solution-phase excitation energies from the Kodak dye-laser catalog (solution $v_{\text {vert }}$ results in Table 2), where methanol or ethanol was used as a solvent. ${ }^{74}$ These results are not directly comparable to our gas-phase values because of differential solvation of the excited state (a different dipole moment from the ground state; see below). Furthermore, the relative excitation energies for different coumarins will also not be directly comparable to our gas-phase values since the ground- and excited-state dipole moment differences vary between coumarins. Nevertheless, the PBE0/TDDFT results do a reasonable job of reproducing the relative excitation energies for these systems. We considered whether the basic ethanol used for the C343 spectra ${ }^{3,4,22}$ might deprotonate C343 and if the experimental spectrum represented that anion of this system. PBE0/TDDFT calculations place the gas-phase $S_{1}$ vertical transition for the $\mathrm{C} 343$ anion at $2.31 \mathrm{eV}$, which is much lower than that observed experimentally. Thus, the calculated results are consistent with the solution spectrum for neutral C343 unless solvation imparts an unexpected blue shift greater than $1 \mathrm{eV}$ for the anion spectrum.

In Table 3, we present dipole moment differences between the $S_{0}$ and $S_{1}$ states of the four coumarins. Since in most experimental techniques one obtains the difference directly, we tabulate it here. In the Supporting Information, we present $S_{1}$ dipole moments, but the experimental values are often based on the assumption of collinearity of the ground- and excitedstate dipole moments since in most experimental results the relative angle is not obtained. Quoting the dipole moment difference also minimizes the effects of incorrect ground-state dipole moments on comparisons of $\mathrm{S}_{1}$ dipole moments.

We find the PBE0/TDDFT dipole moment differences to be $5 \pm 1 \mathrm{D}$ for each of the four molecules. C152 and C153 have the largest dipole moment differences, whereas C102 and C343 have the smallest differences. The CIS values are clustered around 3.4 D within $0.6 \mathrm{D}$ of each other, with a similar grouping of coumarins with the largest and smallest differences. The previous AM1 results tend to be higher than our PBE0/TDDFT values, with the exception of $\mathrm{C} 102$, which is considerably below the PBE0/TDDFT result.

$\mathrm{C} 153$ has been treated previously using the widest array of theoretical techniques, and several past values for the dipole 
TABLE 4: Vertical $S_{1}-S_{2}$ Energy Separation (eV) for the Ground-State Equilibrium Geometry

\begin{tabular}{lllll}
\hline \multicolumn{1}{c}{ method } & $\mathrm{C} 152$ & \multicolumn{1}{c}{${\mathrm{C} 153^{a}}^{a}$} & $\mathrm{C} 102$ & $\mathrm{C} 343$ \\
\hline INDO S/CI & 0.35 & 0.32 & 0.37 & 0.31 \\
PBE0/TDDFT & 0.69 & $0.57 / 0.62$ & 0.53 & 0.59 \\
CIS & 0.85 & $0.78 / 0.83$ & 0.62 & 0.86
\end{tabular}

${ }^{a}$ The first entry is for the syn structure, and the second is for the anti structure.

TABLE 5: Ionization Potential Data (eV)

\begin{tabular}{lcccc}
\hline \multicolumn{1}{c}{ source } & C152 & \multicolumn{1}{c}{ C153 } & C102 & C343 \\
\hline UV-PES $^{83}$ & & & 7.07 & \\
UV $^{84}$ & & $7.21 / 7.16$ & & \\
PBE0/vertical & 7.52 & $7.16 / 7.08^{a}$ & 6.88 & 7.36 \\
PBE0/adiabatic & 7.44 & $7.02 / 6.99^{a}$ & 6.73 & 7.23
\end{tabular}

${ }^{a}$ First entry is for the syn structure, and the second is for the anti structure.

moment difference are presented in Table 3. The largest dipole moment difference was obtained using Grimme's DFT S/CI and is near $15 \mathrm{D}$. Extension to the DFT/MRSDCI drops the difference to $10.7 \mathrm{D}$, but this is still the next-largest estimate of the dipole difference. The other methods give values for the dipole moment that differ in the range of 3.7-7.5 D. Our PBE0/ TDDFT and CIS values fall within this range.

The first five entries in Table 3 comprise experimental estimates of the $S_{1}$ state dipole moment differences for each of the four coumarins. Solvent-induced solute polarization, the solvent model employed, the size of the solute cavity used in analysis of the experimental data, and possible cavity-field effects all complicate the direct comparison with any of the theoretical dipole moments. The most complete experimental treatment of these effects was done by Chowdhury et al. ${ }^{75}$ for C153. Their results suggest that the CIS results of Matyushov and Newton ${ }^{76}$ and our CIS results are closest to the experimental value for $\mathrm{C} 153$. However, to the extent that the cavity and/or reaction-field effects are overestimated, these are lower bounds on the dipole difference. The similarity of the calculated dipole moments for the various coumarins considered here suggests that the experimental dipole moment differences for $\mathrm{C} 152, \mathrm{C} 102$, and $\mathrm{C} 343$ should not be very different from the experimental value for $\mathrm{C} 153$. Interestingly, this is borne out by comparisons within any single experimental study, even though a comparison of the results between experimental studies shows a wide variation in the dipole moment differences for any one coumarin.

Energy differences between the $\mathrm{S}_{1}$ and $\mathrm{S}_{2}$ states are presented in Table 4, computed at the $S_{0}$ equilibrium geometry. The $S_{2}$ state lies about $0.5 \mathrm{eV}$ above the $\mathrm{S}_{1}$ state, independent of the choice of coumarin or method. This is fully consistent with our previous results for $\mathrm{C} 151$ and $\mathrm{C} 120$. This means that by carefully selecting the laser excitation wavelength to be on the red edge of the absorption band, one can preferentially excite the $S_{1}$ state, avoiding the $S_{2}$ state.

A number of studies of the low-lying cation states of coumarins have been made. In Table 5, we present vertical and adiabatic ionization potentials (IPs) calculated using the PBE0 functional. Excellent agreement is obtained with the experimental results for $\mathrm{C} 102$ and $\mathrm{C} 153$. Furthermore, the PBE0 results for the two conformers of $\mathrm{C} 153$ (syn and anti) are consistent with the relative energies obtained experimentally. We expect that the relative IPs among the four coumarins should be good estimates of actual relative IPs for these systems. The adiabatic IPs indicate that there is little energy (about $0.1 \mathrm{eV}$ ) associated with the change in geometry between the neutral and ionic ground states in all four coumarins.
TABLE 6: Comparisons of Various Properties for C153 Structures $^{a}$

\begin{tabular}{lcc}
\hline \multicolumn{1}{c}{ property } & syn & anti \\
\hline$\Delta E_{\mathrm{gs}}(\mathrm{eV})$ & 0.0 & 0.0113 \\
$\mu(\mathrm{D})$ & 7.24 & 7.61 \\
$\mathrm{IP}_{\text {vert }}(\mathrm{eV})$ & 7.16 & 7.08 \\
$\mathrm{IP}_{\text {adiabatic }}(\mathrm{eV})$ & 7.08 & 6.99 \\
$\Delta E_{\mathrm{S} 0-\mathrm{S} 1}(\mathrm{eV})$ & 3.41 & 3.41 \\
$\Delta E_{\mathrm{S} 0-\mathrm{S} 2}(\mathrm{eV})$ & 3.98 & 4.03 \\
$\mu_{\mathrm{S} 1}(\mathrm{D})$ & 12.8 & 13.1
\end{tabular}

${ }^{a}$ PBE0-based results at B3LYP/6-311G(d,p) minima.

We compare the full range of properties for the syn and anti conformers of C153 in Table 6 (see Scheme 2). The relative energies suggest that the syn conformer is the ground state of the system but that the anti conformer will also be populated at room temperature. (The two anti conformers are isoenergetic given the local planar symmetry with respect to the $\mathrm{CF}_{3}$ group.) As shown in Table 5, the syn conformer is somewhat more difficult to ionize than the anti. The $S_{0}$ and $S_{1}$ dipole moments are about $0.3 \mathrm{D}$ larger for the anti conformer, but the vertical excitation energy was identical for the two conformers. Given these similarities, it is clear that the various conformers of C153 should behave similarly as solvatochromic probes.

Because of the presence of the carboxyl group on C343, there are several ground-state protonation conformers that one must consider. We examined the five possibilities shown in Scheme 3 , with selected results given in Table 7 . The global minimum for the ground state of the system has hydrogen on the carboxyl group, forming an intramolecular $\mathrm{H}$ bond with the nearby carbonyl group (A. carboxyl-H: H-bond). This is the only C343 structure for which a frequency analysis was performed, and it was found to be a true minimum. Breaking the $\mathrm{H}$ bond in structure $\mathrm{A}$ by rotating the hydroxyl bond torsional angle $180^{\circ}$ yields structure B and raises the ground-state energy by 0.253 $\mathrm{eV}$. Somewhat higher in energy is the structure with the carboxyl group rotated (C. carboxyl-H: no H-bond2). The zwitterionic structures with the hydrogen transferred to the carbonyl are both significantly higher in energy (either in the gas phase or in PCM chloroform), and we were unable to locate a minimum for the carbonyl-H: H-bond structure (D). Optimizations begun near this point collapsed to the global minimum. The structure used in Table 7 for the carbonyl-H: H-bond (D) was obtained from the optimized carbonyl-H: no $\mathrm{H}$-bond structure $(\mathrm{E})$ by rotating $180^{\circ}$ about the $\mathrm{C}-\mathrm{C}-\mathrm{O}-\mathrm{H}$ dihedral angle. Whereas the carbonyl-H: no $\mathrm{H}$-bond structure $(\mathrm{E})$ is the global minimum for the zwitterionic case, its energy is higher than the rotated structure, as seen in Table 7. Solvation brings the three local minima somewhat closer in energy but does not change their relative ordering. The $S_{1}$ state vertical excitation energies vary significantly for these four structures. For ease of comparison of the $S_{1}$ state energies in the five geometries, we also present excitation energies relative to the ground state at the carboxyl$\mathrm{H}$ : H-bond structure (A). The largest energy difference among the five geometries for the $S_{1}$ state is only $0.33 \mathrm{eV}$, which is significantly smaller than the 2.29-eV range for the ground state. Energies for the $\mathrm{S}_{1}$ state carboxyl $\mathrm{H}$ : $\mathrm{H}$-bond (A) and carbonyl $\mathrm{H}$ : H-bond (D) structures are close enough that one might imagine that solvation could reverse their order. However, the $\mathrm{S}_{1}$ state dipole moment is considerably smaller in the carbonyl structure than in the carboxyl structure. Thus, one expects that solvation will lead to a greater energy separation of these two conformers. 


\section{SCHEME 3}

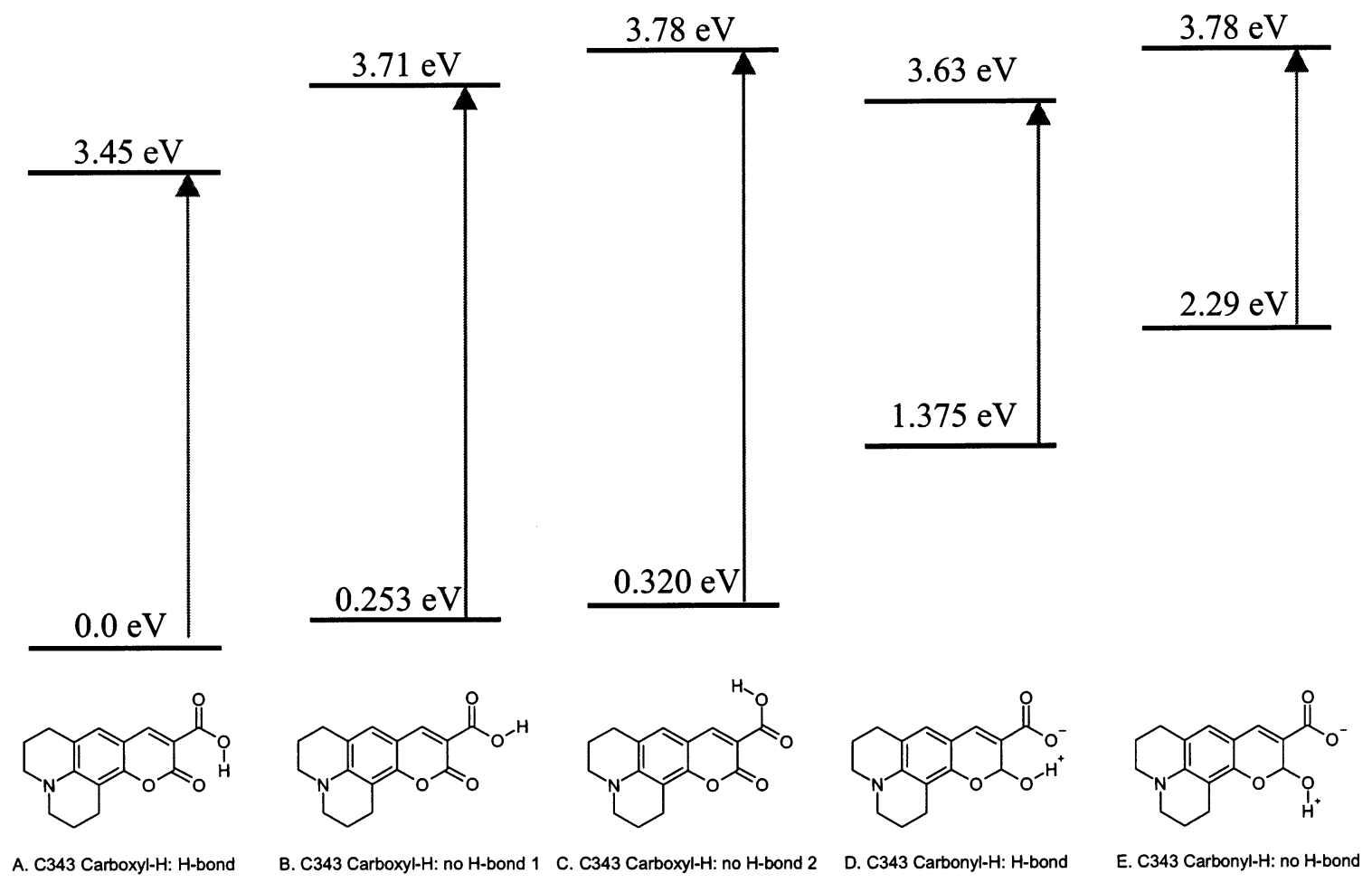

TABLE 7: Comparisons of Various Properties for C343 Structures $^{a}$

\begin{tabular}{|c|c|c|c|c|c|}
\hline property & $\begin{array}{c}\text { A. } \\
\text { carboxyl-H: } \\
\text { H-bond }\end{array}$ & $\begin{array}{c}\text { B. } \\
\text { carboxyl-H: } \\
\text { no H-bond1 }\end{array}$ & $\begin{array}{c}\text { C. } \\
\text { carboxyl-H: } \\
\text { no H-bond2 }\end{array}$ & $\begin{array}{c}\text { D. } \\
\text { carbonyl-H: } \\
\text { H-bond }^{b}\end{array}$ & $\begin{array}{c}\text { E. } \\
\text { carbonyl-H: } \\
\text { no H-bond }\end{array}$ \\
\hline$\Delta E_{\mathrm{gs}}(\mathrm{eV})$ & 0.0 & 0.253 & 0.320 & 1.38 & 2.29 \\
\hline$\mu(\mathrm{D})$ & 11.76 & 8.16 & 9.69 & 16.23 & 19.09 \\
\hline$\Delta E_{\mathrm{gs}}^{\text {solv }}(\mathrm{eV})^{c}$ & 0.0 & & 0.278 & & 1.71 \\
\hline $\mathrm{IP}_{\text {vert }}(\mathrm{eV})$ & 7.36 & & & & \\
\hline $\mathrm{IP}_{\text {adiabatic }}(\mathrm{eV})$ & 7.23 & & & & \\
\hline$\Delta E_{\mathrm{S} 0-\mathrm{S} 1}(\mathrm{eV})^{d}$ & 3.45 & 3.45 & 3.46 & 2.26 & 1.49 \\
\hline$\Delta E_{\mathrm{S} 0-\mathrm{S} 2}(\mathrm{eV})^{d}$ & 4.04 & 3.91 & 3.90 & 2.74 & 1.72 \\
\hline$\Delta E_{\mathrm{S} 0 \min -\mathrm{S} 1}(\mathrm{eV})^{e}$ & 3.45 & 3.71 & 3.78 & 3.63 & 3.78 \\
\hline$\mu_{\mathrm{S} 1}(\mathrm{D})$ & 15.8 & & & 5.5 & \\
\hline
\end{tabular}

${ }^{a}$ PBE0-based results at B3LYP/6-311G(d,p) minima. ${ }^{b}$ Obtained by rotating about the $\mathrm{C}-\mathrm{C}-\mathrm{O}-\mathrm{H}$ dihedral angle for the carbonyl-H: no $\mathrm{H}-$ bond structure-no ground-state minimum was located for this structure. ${ }^{c}$ Based the PCM solvation model, in chloroform. ${ }^{d}$ Vertical excitation energy at the given geometry. ${ }^{e}$ Energy of the $\mathrm{S}_{1}$ state at the given geometry relative to that of the ground state at the carboxyl-H: H-bond ground-state structure.

\section{Discussion}

The above results support and reinforce many of the conclusions we drew in our previous study of coumarins 151 and 120. In particular, we find that DFT tends to overestimate the groundstate dipole moment of the coumarins modestly (by <20\%) and that values for either INDO or MP2 dipole moments tend to be closer to the experimental results for these systems. For the excited states, TDDFT based on the PBE0 functional provides exceptional accuracy for the vertical transition energy to the $S_{1}$ state and yields excellent excitation energy trends for coumarins in nonpolar solvent. INDO S/CI also does a good job of reproducing the excitation energies and their variation among the four coumarins treated here. TDDFT predicts that the $\mathrm{S}_{1}-$ $\mathrm{S}_{2}$ gap is on the order of $0.5 \mathrm{eV}$ for the ground-state equilibrium geometry. Our TDDFT $\mathrm{S}_{0}-\mathrm{S}_{1}$ dipole moment differences for $\mathrm{C} 152$ and $\mathrm{C} 153$ fall in the middle of the various experimental estimates and are generally about $1 \mathrm{D}$ higher than the CIS values. The CIS value for $\mathrm{C} 153$ is close to the value that yields the best agreement with the experimental results of Chowdhury et al. ${ }^{75}$ when corrected for reaction and cavity fields. However, given the difficulty in accurately defining solvent cavities for these systems and the restriction to dipolar fields, uncertainties of $\pm 1 \mathrm{D}$ may not be unreasonable. Certainly the TDDFT results do not suggest that the extreme dipole moment differences are likely. Finally, as was found previously, the DFT ionization potentials are in excellent agreement with experiment and support the experimental assignment of the different C153 conformers.

Because of the julolidyl ring structure in $\mathrm{C} 102, \mathrm{C} 153$, and C343, we were also able to examine the sensitivity of the electronic properties to the choice of syn or anti conformations at the julolidyl nitrogen. The difference between the two conformers was examined in detail for $\mathrm{C} 153$. We found that the syn conformer was more stable than the anti conformer by $0.011 \mathrm{eV}$ but that their excitation energies, dipole moments, and dipole moment differences were similar enough that they should be indistinguishable in solution. However, in the gas phase, their IPs are distinct enough to allow for easy assignment of the two species. 
C343 affords an additional level of complexity because of the presence of the carboxylic acid group. Our investigation of the five structures shown in Scheme 3 indicates that only one is thermally accessible in the ground state but that all five are within $0.33 \mathrm{eV}$ of each other on the $\mathrm{S}_{1}$ surface. In addition, the ground-state dipole moments are quite different in these various structures and thus should yield different solvent stabilization energies. Nevertheless, we find that the addition of a solvent reaction-field model neither reorders nor dramatically alters the energy difference between the two lowest-energy structures for C343.

Taken together, our results suggest that TDDFT is an accurate and powerful tool for the examination of the ground and excited states of coumarins. In particular, the accuracy achieved for excitation energies and ionization potentials is good enough to aid in assigning experimental spectra for these systems. Furthermore, dipole moment differences between ground and excited states are comparable to those obtained by other methods and are certainly found to be within the spread of experimental values (where available). These results suggest that where charge-transfer states (between localized diabatic states) are not involved, TDDFT yields impressive results for molecular properties

\section{Conclusions}

Our results indicate that TDDFT is a powerful tool for treating the spectroscopy and properties of the $S_{1}$ state of the coumarins. Excitation energies, dipole moment differences, and ionization potentials are in good agreement with experiment where comparisons can be made. We find no evidence for excitedstate proton transfer in $\mathrm{C} 343$ and see little spectroscopic difference between the two possible julolidyl conformers of $\mathrm{C} 153$, especially in room-temperature solutions. Finally, we conclude the INDO S/CI is also a reliable tool for the treatment of the spectra and properties of these systems.

Acknowledgment. R.J.C. and E.W.C. acknowledge independent financial support from the donors of the Petroleum Research Fund. R.J.C. gratefully acknowledges support from the Rutgers University Nanochemistry Consortium and from the National Science Foundation (CHE-9731634).

Supporting Information Available: Calculations on coumarins, state dipole moments, molecular geometries, and standard and input orientations. This material is available free of charge via the Internet at http://pubs.acs.org.

\section{References and Notes}

(1) Dye Lasers, 3rd ed.; Schäfer, F. P., Ed.; Springer-Verlag: Berlin, 1990; Vol. 1, p 244.

(2) Horng, M. L.; Gardecki, J. A.; Maroncelli, M. J. Phys. Chem. A 1997, 101, 1030-1047.

(3) Riter, R. E.; Willard, D. M.; Levinger, N. E. J. Phys. Chem. B 1998, 102, 2705-2714.

(4) Pant, D.; Riter, R. E.; Levinger, N. E. J. Chem. Phys. 1998, 109, 9995-10003.

(5) Sarkar, N.; Das, K.; Datta, A.; Das, S.; Bhattacharyya, K. J. Phys. Chem. 1996, 100, 10523-10527.

(6) Datta, A.; Pal, S. K.; Mandal, D.; Bhattacharyya, K. J. Phys. Chem. $B$ 1998, 102, 6114-6117.

(7) Pal, S. K.; Sukul, D.; Mandal, D.; Sen, S.; Bhattacharyya, K. J. Phys. Chem. B 2000, 104, 2613-2616.

(8) Castner, E. W., Jr.; Bagchi, B.; Maroncelli, M.; Webb, S. P.; Ruggiero, A. J.; Fleming, G. R. Ber. Bunsen-Ges. Phys. Chem. 1988, 92 , $363-372$.

(9) Vajda, S.; Jimenez, R.; Rosenthal, S. J.; Fidler, V.; Fleming, G. R.; Castner, E. W., Jr. J. Chem. Soc., Faraday Trans. 1995, 91, 867-873. 2376.

(10) Shirota, H.; Castner, E. W., Jr. J. Chem. Phys. 2000, 112, 2367-

(11) Frauchiger, L.; Shirota, H.; Uhrich, K. E.; Castner, E. W., Jr. J. Phys. Chem. B 2002, 106, 7463-7468.

(12) Castner, E. W., Jr.; Maroncelli, M.; Fleming, G. R. J. Chem. Phys. 1987, 86, 1090-1097.

(13) Maroncelli, M.; Fleming, G. R. J. Chem. Phys. 1987, 86, 62216239.

(14) Nagarajan, V.; Brearley, A. M.; Kang, T. J.; Barbara, P. F. J. Chem. Phys. 1987, 86, 3183-3196.

(15) Kahlow, M. A.; Kang, T. J.; Barbara, P. F. J. Chem. Phys. 1988, $88,2372-2378$

(16) Jarzeba, W.; Walker, G. C.; Johnson, A. E.; Barbara, P. F. Chem. Phys. 1991, 152, 57-68.

(17) Jimenez, R.; Fleming, G. R.; Kumar, P. V.; Maroncelli, M. Nature (London) 1994, 369, 471-473.

(18) Rosenthal, S. J.; Jimenez, R.; Fleming, G. R.; Kumar, P. V.; Maroncelli, M. J. Mol. Liq. 1994, 60, 25-56.

(19) Horng, M. L.; Gardecki, J. A.; Papazyan, A.; Maroncelli, M. J. Phys. Chem. 1995, 99, 17311-17337.

(20) Pal, H.; Nagasawa, Y.; Tominaga, K.; Kumazaki, S.; Yoshihara, K. J. Chem. Phys. 1995, 102, 7758-7760.

(21) Lee, B. J.; Diken, E. L.; Wiewior, P. P.; Castner, E. W., Jr. J. Phys. Chem. B, to be submitted for publication.

(22) Riter, R. E.; Kimmel, J. R.; Undiks, E. P.; Levinger, N. E. J. Phys Chem. B 1997, 101, 8292-8297.

(23) Sarkar, N.; Datta, A.; Das, S.; Bhattacharyya, K. J. Phys. Chem. 1996, 100, 15483-15486.

(24) Pant, D.; Levinger, N. E. Chem. Phys. Lett. 1998, 292, 200-206.

(25) Das, K.; Sarkar, N.; Das, S.; Datta, A.; Bhattacharyya, K. Chem. Phys. Lett. 1996, 249, 323-328.

(26) Changenet-Barret, P.; Choma, C. T.; Gooding, E. F.; DeGrado, W. F.; Hochstrasser, R. M. J. Phys. Chem. B 2000, 104, 9322-9329.

(27) Bart, E.; Meltsin, A.; Huppert, D. J. Phys. Chem. 1994, 98, 1081910823

(28) Bart, E.; Meltsin, A.; Huppert, D. J. Phys. Chem. 1994, 98, 32953299.

(29) Seidel, C. A. M.; Schulz, A.; Sauer, M. H. M. J. Phys. Chem. 1996, $100,5541-5553$

(30) Jones, G. I.; Griffin, S. F.; Choi, C. -y.; Bergmark, W. R. J. Org Chem. 1984, 49, 2705-2708.

(31) Jones, G., II; Jackson, W. R.; Choi, C. Y.; Bergmark, W. R. J. Phys. Chem. 1985, 89, 294-300.

(32) Yoshihara, K. Adv. Chem. Phys. 1999, 107, 371-402.

(33) Shirota, H.; Pal, H.; Tominaga, K.; Yoshihara, K. J. Phys. Chem. A 1998, 102, 3089-3102.

(34) Pal, H.; Shirota, H.; Tominaga, K.; Yoshihara, K. J. Chem. Phys 1999, 110, 11454-11465.

(35) Nad, S.; Pal, H. J. Photochem. Photobiol., A 2000, 134, 9-15.

(36) Nad, S.; Pal, H. J. Phys. Chem. A 2000, 104, 673-680.

(37) Castner, E. W., Jr.; Kennedy, D.; Cave, R. J. J. Phys. Chem. A 2000, 104, 2869-2885.

(38) Rehm, J. M.; McLendon, G. L.; Nagasawa, Y.; Yoshihara, K.; Moser, J.; Gratzel, M. J. Phys. Chem. 1996, 100, 9577-9578.

(39) Ghosh, H. N.; Asbury, J. B.; Lian, T. J. Phys. Chem. B 1998, 102 $6482-6486$

(40) Ghosh, H. N.; Asbury, J. B.; Lian, T. PINSA-A: Proc. Indian Nat Sci. Acad., Part A 2000, 66, 177-197.

(41) Murakoshi, K.; Yanagida, S.; Capel, M.; Castner, E. W., Jr. Interfacial Electron-Transfer Dynamics of Photosensitized Zinc Oxide Nanoclusters. In Nanostructured Materials: Clusters, Thin Films, and Composites; Shalaev, V., Moskovits, M., Eds.; American Chemical Society: Washington, DC, 1997; Vol. 679, pp 221-238.

(42) Matsumoto, M.; Murakoshi, K.; Wada, Y.; Yanagida, S. Chem Lett. 2000, 938-939.

(43) Druzhinin, S. I.; Bursulaya, B. D.; Uzhinov, B. M. J. Photochem Photobiol., A 1995, 90, 53-56.

(44) Goryaeva, E. M.; Shablya, A. V. Opt. Spektrosk. 1997, 83, 853859.

(45) Chudoba, C.; Nibbering, E. T. J.; Elsaesser, T. Phys. Rev. Lett. 1998, 81, 3010-3013.

(46) Tschirschwitz, F.; Nibbering, E. T. J. Chem. Phys. Lett. 1999, 312, 169-177.

(47) Chudoba, C.; Nibbering, E. T. J.; Elsaesser, T. J. Phys. Chem. A 1999, $103,5625-5628$

(48) Nibbering, E. T. J.; Tschirschwitz, F.; Chudoba, C.; Elsaesser, T. J. Phys. Chem. A 2000, 104, 4236-4246.

(49) Cave, R. J.; Burke, K.; Castner, E. W., Jr. J. Phys. Chem. A 2002, 106, 9294-9305.

(50) Runge, E.; Gross, E. K. U. Phys. Rev. Lett. 1984, 52, 997. 
(51) Casida, M. E. Time-Dependent Density Functional Response Theory for Molecules. In Recent Advances in Density Functional Theory Methods, Part I; Chong, D. P., Ed; World Scientific: Singapore, 1995; pp 155193.

(52) Casida, M. E. Time-Dependent Density Functional Response Theory of Molecular Systems: Theory, Computational Methods, and Functionals. In Recent Developments and Applications of Modern Density Functional Theory; Seminario, J. M., Ed.; Elsevier: Amsterdam, 1996; p 391.

(53) Finley, J.; Malmqvist, P. A.; Roos, B. O.; Serrano-Andres, L. Chem. Phys. Lett. 1998, 288, 299-306.

(54) Roos, B. O.; Andersson, K.; Fulscher, M. P.; Malmqvist, P. A.; SerranoAndres, L.; Pierloot, K.; Merchan, M. Adv. Chem. Phys. 1996, 93 219-331.

(55) Roos, B. O. Acc. Chem. Res. 1999, 32, 137-144.

(56) Zerner, M. C.; Loew, G. H.; Kirchner, R. F.; Mueller-Westerhoff,

U. T. J. Am. Chem. Soc. 1980, 102, 589.

(57) Zerner, M. C. Zindo Electronic Structure Theory Code, 3.7 ed.; Molecular Simulations, Inc.: La Jolla, CA, 1991.

(58) Palmer, P. M.; Chen, Y.; Topp, M. R. Chem. Phys. Lett. 2000, $318,440-447$.

(59) Pryor, B. A.; Palmer, P. M.; Andrews, P. M.; Berger, M. B.; Topp,

M. R. J. Phys. Chem. A 1998, 102, 3284-3292.

(60) Perdew, J. P.; Ernserhof, M.; Burke, K. J. Chem. Phys. 1996, 105, 9982.

(61) Krishnan, R.; Binkley, J. S.; Seeger, R.; Pople, J. A. J. Chem. Phys. 1980, 72,650 .

(62) McLean, A. D.; Chandler, G. S. J. Chem. Phys. 1980, 72, 5639

(63) Becke, A. D. J. Chem. Phys. 1993, 98, 5648.

(64) Cossi, M.; Barone, V.; Cammi, R.; Tomasi, J. Chem. Phys. Lett. 1996, 255, 327.

(65) Miertus, S.; Scrocco, E.; Tomasi, J. Chem. Phys. 1981, 55, 117.

(66) Miertus, S.; Tomasi, J. Chem. Phys. 1982, 65, 239.

(67) Frisch, M. J.; Trucks, G. W.; Schlegel, H. B.; Scuseria, G. E.; Robb, M. A.; Cheeseman, J. R.; Zakrzewski, V. G.; Montgomery, J. A., Jr.; Stratmann, R. E.; Burant, J. C.; Dapprich, S.; Millam, J. M.; Daniels, A. D.; Kudin, K. N.; Strain, M. C.; Farkas, O.; Tomasi, J.; Barone, V.; Cossi, M.; Cammi, R.; Mennucci, B.; Pomelli, C.; Adamo, C.; Clifford, S.; Ochterski, J.; Petersson, G. A.; Ayala, P. Y.; Cui, Q.; Morokuma, K.; Malick,
D. K.; Rabuck, A. D.; Raghavachari, K.; Foresman, J. B.; Cioslowski, J.; Ortiz, J. V.; Stefanov, B. B.; Liu, G.; Liashenko, A.; Piskorz, P.; Komaromi, I.; Gomperts, R.; Martin, R. L.; Fox, D. J.; Keith, T.; Al-Laham, M. A.; Peng, C. Y.; Nanayakkara, A.; Gonzalez, C.; Challacombe, M.; Gill, P. M. W.; Johnson, B. G.; Chen, W.; Wong, M. W.; Andres, J. L.; Head-Gordon, M.; Replogle, E. S.; Pople, J. A. Gaussian 98, revisions A.9 and A.11; Gaussian, Inc.: Pittsburgh, PA, 1998.

(68) Dewar, M. J. S.; Zoebisch, E. G.; Healy, E. F. J. Am. Chem. Soc. 1985, 107, 3902-3909.

(69) Stewart, J. J. P. J. Comput. Chem. 1989, 10, 209-220.

(70) Stewart, J. J. P. J. Comput. Chem. 1989, 10, 221

(71) Moylan, C. R. J. Phys. Chem. 1994, 98, 13513-13516.

(72) Muhlpfordt, A.; Schanz, R.; Ernsting, N. P.; Farztdinov, V.; Grimme, S. Phys. Chem. Chem. Phys. 1999, 1, 3209-3218.

(73) McCarthy, P. K.; Blanchard, G. J. J. Phys. Chem. 1993, 97, 1220512209

(74) Duarte, F.; Birge, R. R. Kodak Laser Dye Catalog; Eastman Kodak, Inc.: Rochester, NY, 1989.

(75) Chowdhury, A.; Locknar, S. A.; Premvardhan, L. L.; Peteanu, L. A. J. Phys. Chem. A 1999, 103, 9614-9625.

(76) Matyushov, D. V.; Newton, M. D. J. Phys. Chem. A 2001, 105, 8516-8532.

(77) Rechthaler, K.; Koehler, G. Chem. Phys. 1994, 189, 99-116.

(78) Moog, R. S.; Davis, W. W.; Ostrowski, S. G.; Wilson, G. L. Chem Phys. Lett. 1999, 299, 265-271.

(79) Ernsting, N. P.; Asimov, M.; Schaefer, F. P. Chem. Phys. Lett. 1982 91, 231-235

(80) Samanta, A.; Fessenden, R. W. J. Phys. Chem. A 2000, 104, 85778582 .

(81) Ravi, M.; Soujanya, T.; Samanta, A.; Radhakrishnan, T. P. J. Chem. Soc., Faraday Trans. 1995, 91, 2739-2742.

(82) Smirnov, S. N.; Braun, C. L. Rev. Sci. Instrum. 1998, 69, 28752887.

(83) Novak, I.; Kovac, B. J. Electron Spectrosc. Relat. Phenom. 2000, $113,9-13$.

(84) Pryor, B. A.; Palmer, P. M.; Chen, Y.; Topp, M. R. Chem. Phys. Lett. 1999, 299, 536-544. 